\title{
Estudio del nivel de diversión generado por la aplicación de un programa de entrenamiento técnico y otro táctico en futbolistas jóvenes
}

\author{
Enjoy level research generated by technical and tactical \\ training programs in young soccer players
}

\section{Estudo do nível de diversão gerado pela aplicação de um programa de treinamento técnico e outro tático em jovens jogadores de futebol}

\author{
Javier Sánchez Sánchez*, José Ma Yagüe Cabezón** y Olga Molinero González** \\ *Universidad Pontificia de Salamanca y Universidad de León ${ }^{* *}$
}

Resumen: La diversión debe ser un valor derivado de las estrategias desarrolladas durante la etapa de aprendizaje deportivo. Por este motivo pretendemos determinar qué modelo de enseñanza-entrenamiento del fútbol puede generar mayor disfrute en jugadores jóvenes. Para ello 101 futbolistas de edades entre 6 y 12 años, han sido consultados sobre la diversión asociada a un entrenamiento técnico, a situaciones artificiales, descargadas de cualquier dificultad cognitiva con el propósito de mecanizar un gesto determinado, y a otro táctico, a través de la participación en tareas globales que inciden en los procesos de percepción, decisión y en el mecanismo de ejecución del jugador; por medio de la administración del Cuestionario de diversión con el entrenamiento del fútbol, que fue generado a partir de la herramienta de Duda y Nicholls (1992). Los resultados del trabajo muestran que los jugadores se divierten con ambos programas, si bien, es el entrenamiento basado en juegos de aplicación táctica el que genera mayor disfrute y con el que los futbolistas pasan mejor el tiempo de práctica. Nuestro programa de entrenamiento táctico, que es el que concede más libertad al jugador para resolver los problemas derivados de su intervención sería el que más aumenta la motivación. Este factor es elemental, ya que una mayor amotivación provocaría niveles de diversión más bajos en los sujetos (Guay, Vallerand y Blanchard, 2000; Carratalá, Guzmán, Carratalá y García, 2006), que constituye una de la principales razones de abandono deportivo en edades tempranas (Blázquez y Batalla, 1995; Molinero, Salguero, Tuero, Álvarez y Márquez, 2006).

Palabras Clave: Diversión, entrenamiento técnico, entrenamiento táctico, fútbol-base.

Abstract: Enjoyment should be a value derived from the strategies developed during the sport learning stage. For this reason, we aim to determine which model of teaching-training soccer could engender greater enjoyment in young players. 101 players aged between 6 and 12 years have been consulted about the fun associated with technical training, defined as artificial situations discharged of any cognitive impairment in order to mechanize a particular ability; and tactical training through by taking part in global tasks that affect the processes of perception, decision and performance of the athlete. We administered the Questionnaire of Fun with Soccer Training, which was generated from Duda and Nicholls' inventaire (1992). The results of the study show that players had fun from both programs, although training based on tactical games generates greater enjoyment and players spend more practice time on it. Our tactical training program, which gives more freedom to solve problems, would be higher on motivation levels. This factor is fundamental, because higher levels of motivation make fun decrease (Guay, Vallerand \& Blanchard, 2000; Carratalá, Guzman, Carratalá \& Garcia, 2006), and this is the main reason why children and adolescents drop out sports (Blázquez \& Batalla, 1995; Molinero, Salguero, Tuero, Álvarez \& Márquez, 2006)

Key words: Fun, Technical training, Tactical training, soccer learning. Resumo: A diversão deve ser um valor derivado das estratégias desenvolvidas durante a etapa de aprendizagem esportiva. Por esse motivo pretendemos determinar qual modelo de ensino-treino de futebol pode gerar maior satisfação em jovens jogadores de futebol. Participaram 101 jogadores de futebol entre 6 e 12 anos de idade, foram consultados temas sobre a diversão associada a um treinamento técnico, situaçóes artificiais, descargas de qualquer dificuldade cognitiva com o propósito de mecanizar um gesto determinado e temas relacionados ao tático como a participação em tarefas globais que incidem nos processo de percepção, decisão no mecanismo de execução do jogador; por meio da administração do questionário de diversấo com o treinamento de futebol, que foi criado a partir da ferramenta de Duda y Nicholl (1992). Os resultados do estudo mostram que os jogadores se divertem com ambos os programas, sendo que o treinamento baseado em jogo de aplicação tática o que gera mais satisfação e o qual os jogadores se divertem mais durante as horas de prática. Nosso programa de treinamento tático concede mais liberdade ao jogador para resolver os problemas derivados de sua intervenção é o que mais aumenta a motivação. Esse fator seria fundamental já que com uma falta de motivação provocaria níveis de diversão mais baixos nos sujeitos (Guay, Vallerand y Blanchard, 2000; Carratalá, Guzmán, Carratalá y García, 2006), e constitue uma da principais razoes do abandono esportivo em idades precoces(Blázquez y Batalla, 1995; Molinero, Salguero, Tuero, Álvarez y Márquez, 2006).

Palavras chave: Diversão, treinamento técnico, treinamento tático, futebol de base.
Dirección para correspondencia
Javier Sánchez Sánchez
C/ Henry Collet 52-70.37007. Salamanca
jsanchezsa@upsa.es 


\section{Introducción}

Los jóvenes que aceptan la práctica deportiva como una tarea más dentro de su actividad diaria, agradecen que los procedimientos que dan forma a sus entrenamientos les permitan disfrutar con lo que hacen. Esta cualidad ligada al entrenamiento es de suma importancia, pues los sujetos que se inician en el deporte, consideran la diversión como uno de los impulsos que refuerzan su práctica (Valero y Latorre, 1998). La diversión es una de las causas que hacen que un niño se involucre en su actividad y su ausencia puede considerarse como una de las razones por las que se abandona la práctica de actividad física (Ewing y Seefeldt, 1988, citados en Garn y Cothran, 2006). Según esto, es evidente que el factor diversión, debería ser un elemento altamente considerado entre todos aquellos técnicos deportivos que desarrollan su labor durante las etapas de formación. Estos profesionales, necesitan conocer las estrategias que convierten la participación de los jugadores en una práctica agradable.

Dentro del marco de la enseńanza-entrenamiento de un deporte, para hacer disfrutar al practicante, se debe utilizar el juego como recurso protagonista dentro de las sesiones (Arias, 2008; Duran y Lasierra, 1987; García, 2001; Ponce, 2006; Torres, 2005; Turner y Martinek, 1992; Valero, 2005). Según Devis y Peiró (1995), para asegurar la diversión es obligado prescindir de las tradicionales intervenciones basadas en la técnica, y optar por otras estrategias, que en el ámbito de los deportes colectivos como el fútbol, se sostienen a través del uso de tareas jugadas, que mantienen intactos todos aquellos patrones que configuran la realidad del deporte. El manejo racional y adaptado de este tipo de actividades por parte del entrenador, conseguirá incrementar la especificidad del aprendizaje, asegurando al mismo tiempo la adherencia del futbolista con la práctica deportiva.

Sin embargo, la diversión como valor interesante dentro de la iniciación deportiva, no siempre ha sido respetada dentro de los programas formativos. Tradicionalmente el interés se localizaba sobre el aprendizaje de la técnica, sin reflexionar sobre las consecuencias que generaban los procedimientos empleados en el intento de dotar al futbolista de estas habilidades. El aburrimiento asociado a la repetición extenuante de movimientos (Cárdenas, 2006), estaba justificado como una forma de conseguir un futbolista técnicamente competente. En el intento por formar sujetos hábiles, los contextos empleados para lograr la mecanización de gestos técnicos carecían de la animación necesaria, dentro de un ambiente ejemplarizante que sometía la diversión al rigor de los resultados. Para conseguir estos logros, los ejercicios eran también de base poco estimulantes, por prescindir de elementos como la cooperación, la oposición y la finalización (Alonso y Lago, 2009). Obviar estos valores que ocupan un lugar indiscutible en la realidad de un deporte como el fútbol, supone menos- preciar el deseo del niño por el juego, provocar el desencanto en un participante que comienza a verse dentro de una actividad que no es tan divertida como pensaba (Blázquez y Batalla, 1995) y aumentar la probabilidad de que el participante abandone la práctica deportiva (Molineroet al., 2006).

Ante la sucesión de elementos discordantes vinculados al método tradicional de enseñanza deportiva, comenzó a cuestionarse seriamente la validez de los postulados derivados de la psicología conductista para las modalidades colectivas (Torres, 2004). Todas las prácticas características de los estilos directivos, basadas en las tareas descontextualizadas, monótonas y ajenas a los intereses y opiniones del deportista (Contreras, De la Torre y Velázquez, 2001; Martínez, 2001; Wein, 1995), no tienen cabida dentro del nuevo panorama dedicado al aprendizaje del deporte. En este contexto se defiende un nuevo concepto de aprendizaje, más comprometido con el niño, sostenido sobre la base de la comprensión del juego y convencido de la necesidad de utilizar estrategias atractivas que faciliten la adherencia del sujeto a la práctica deportiva.

El juego debe ser un recurso omnipresente en la formación deportiva infantil (Lasierra, 1990), no sólo para estimular fielmente los preceptos básicos de disciplinas como el fútbol, sino para proteger la naturaleza del niño. Los nuevos métodos basados en la táctica, tienen muy presente que el fútbol es un juego y realmente se aprenderá a jugar cuando se deje hacerlo (Soares y Santana, 2005). Por esto, no se debe tener miedo por utilizar este tipo de actividades, sino que deben estar presentes desde el mismo momento en que el jugador se acerca al deporte, con independencia de su capacidad relativa (Thorpe, 1992). El progreso en la disciplina deportiva, será consecuencia de un procedimiento que genera diversión en los jugadores, quienes además ven incrementada su implicación y motivación hacia el aprendizaje (Sánchez, 1995).

Pensamos que además de las indiscutibles virtudes para el dominio del fútbol, emplear estrategias globales, genera en los niños sentimientos positivos que permitirán mejorar el aprendizaje e integrar la práctica deportiva dentro de sus rutinas diarias. El factor diversión, debe ser uno de los valores asociados a cualquiera de las prácticas presentes en los programas de formación de las diferentes escuelas de fútbol. Siempre que un niño encuentra divertido lo que hace, permanecerá en la actividad, por lo tanto es necesario conocer qué tareas son las más divertidas y las que más aseguran la vinculación permanente del jugador con el deporte.

Investigaciones como las de Allison y Thorpe (1997), Cervelló (1996), Duran y Lasierra (1987), Griffin, Oslin y Mitchell (1995), Méndez (1999), Ponce (2006), Turner (1996) y Valero (2003), trataron de diagnosticar la diversión que provocaba una determinada estrategia de enseñanza-entrena- 
miento. Los resultados de estos estudios son bastante homogéneos, constatando que aquellas maniobras que utilizan el juego, suelen ser más divertidas que las basadas en automatismos y ejercicios vinculados exclusivamente al mecanismo de ejecución.

Con el presente trabajo pretendemos comprobar el grado de diversión asociado a un tipo de entrenamiento, dentro del ámbito de la iniciación deportiva al fútbol. En concreto, en el estudio se ha diseńado un programa basado en ejercicios analíticos, para que el jugador domine el mecanismo de ejecución relacionado con los gestos técnicos básicos presentes en fútbol; y otro programa basado en juegos de aplicación táctica, con los que se busca que el jugador esté en contacto con el balón y experimente la lógica interna del fútbol.

El conjunto de elementos formales (espacio, balón, compañeros, adversario, etc.) que rodean a una y otra estrategia de intervención, repercutirán en la consideración general de los jugadores. Especialmente significativo, puede ser el nivel de diversión experimentado en cada programa, por esta razón el objetivo de nuestro estudio es: comparar las consecuencias de un programa de entrenamiento técnico y otro táctico en el grado de diversión de niños entre 6 y 12 años. En relación a este objetivo, hemos planteado la siguiente hipótesis: los futbolistas que participan en el programa de entrenamiento basado en juegos de aplicación táctica, se divierten más que aquellos que realizan ejercicios analíticos para el aprendizaje de la técnica individual.

\section{Método}

\section{Participantes}

En el estudio han participado 101 nińos de 6 a 12 años, que se integran dentro de las categorías prebenjamín, benjamín y alevín, que configuran la etapa conocida como fútbol-base. Todos participan en las competiciones organizadas por la Delegación Salmantina de Fútbol, actuando dentro de diversos clubes de la provincia: 36 pertenecen a la U.D. Salamanca, 11 militan en el C.D. Navega y 54 juegan en el C.D.U.D. Santa Marta.

A cada equipo se le aplicó un tipo de entrenamiento: técnico o táctico. Las principales características de la muestra están resumidas en la Tabla 1.

Tabla 1. Principales características de la muestra participante en el estudio (Media (m) y Desviación Estándar (Sd).

\begin{tabular}{llcc}
\hline Grupo & $\mathrm{N}$ & Edad $(\mathrm{m})$ & $\mathrm{Edad}(\mathrm{Sd})$ \\
\hline Técnico & 51 & 8 & 2,04 \\
Táctico & 50 & 9,46 & 1,61 \\
\hline
\end{tabular}

\section{Instrumentos}

Se utilizó un diseño experimental con dos grupos consignados a cada tipo de entrenamiento deportivo: grupo técnico y grupo táctico. En la composición de los grupos se contó con equipos completos, teniendo en cuenta un reparto equilibrado de edades y categorías para cada tipo de intervención.

La variable independiente o factor, es el programa de entrenamiento, que queda establecido en dos niveles:

- Programa de entrenamiento técnico. Con este programa se pretende dominar los gestos técnicos específicos del fútbol a través de la participación en ejercicios analíticos. Para conseguir esta finalidad se recurre a situaciones artificiales, descargadas de cualquier dificultad cognitiva con el propósito de mecanizar un gesto determinado. Son ejercicios poco estimulantes, escasamente creativos y que prescinden de la lógica esencial del juego (oponente, compañero, etc.). Se fundamentan en la repetición y el automatismo.

- Programa de entrenamiento táctico. El objetivo de este programa es aprender los fundamentos tácticos del fútbol, a través de la participación en tareas globales que inciden en los procesos de percepción, decisión y en el mecanismo de ejecución del jugador. Se emplean actividades que mejoran en el niño la capacidad de juego. Implica una elevada demanda cognitiva que lleva implícito grandes dosis de compromiso, interés y adherencia a la práctica. Se fundamentan en el ensayo y la indagación.

La variable dependiente es la diversión asociada a cada tipo de entrenamiento, ya que según nuestra hipótesis, cada tipo de intervención generará en el sujeto una impresión diferente. Para conocer la opinión de cada participante en el programa hemos utilizado el Cuestionario de diversión con el entrenamiento del fútbol. Esta herramienta fue construida a partir del Cuestionario de diversión con la práctica deportiva, original de Duda y Nicholls (1992), que fue actualizado al castellano por Cervelló (1996) y posteriormente validado en el trabajo de Cervelló, Escartí y Balagüe (1999). El instrumento original compuesto por 7 ítems, da información sobre la diversión asociada a la práctica deportiva. Este último instrumento fue utilizado en el marco del atletismo por Valero, Conde, Delgado y Conde (2004). En nuestro estudio hemos ajustado las preguntas al ámbito del entrenamiento del fútbol. Para ello hemos prescindido de la palabra deporte e introducido la expresión entrenamiento del fútbol. Además, tal y como se procedió en el estudio referido al atletismo de Valero et al. (2004), sustituimos la escala tipo Likert original que mantenía el rango de respuesta de 0 a 100 , por otra más simple reducida a cuatro niveles ( $\mathrm{Nada}$ es 0 , Poco es 1 , Bastante es 2 y Mucho es 3). 


\section{Procedimiento}

La aplicación de los programas se desarrolló a lo largo de 9 semanas, con la ayuda de los entrenadores de cada equipo. Al finalizar la sesión número 15 cada jugador completó el Cuestionario de diversión con el entrenamiento del fútbol. Los jugadores respondieron a las preguntas de manera autónoma, salvo algunos que por su edad, demandaron la ayuda de los entrenadores.

Para conocer la incidencia de cada programa sobre la diversión se realizó un estudio estadístico, empleando para el tratamiento de los datos el programa SPSS 18.0 para Windows. En primer lugar se calcularon las frecuencias y porcentajes de la información registrada y a continuación, se obtuvo una cifra representativa del valor asignado por la muestra a cada ítem. Esto último, que se conoce con el nombre de indicador sintético de frecuencia, se calcula fijando un valor a cada categoría de respuesta de la Escala Likert empleada en el cuestionario (Nada es 0, Poco es 1, Bastante es 2 y Mucho es 3), a continuación se multiplica el valor por su frecuencia y se divide el resultado por el número de componentes de la muestra.

Una vez conocido el indicador sintético de frecuencia de cada ítem del cuestionario, se compararon los valores con la prueba $t$ de Student para muestras independientes. Este último análisis estuvo precedido por una prueba de contraste de Levene para determinar la homogeneidad de las varianzas, teniendo en cuenta una significación p $>0,05$. En el resto de casos, a efectos de interpretación y análisis de los resultados, asumimos el 95\% como intervalo de confianza, de manera que las diferencias entre grupos son significativas cuando $\mathrm{p}$ $\leq 0,05$, muy significativas cuando $\mathrm{p} \leq 0,01$ y altamente significativas cuando $\mathrm{p} \leq 0,001$.

\section{Resultados}

Los jugadores que han entrenado a través del modelo de entrenamiento basado en la técnica realizan una apreciación general del entrenamiento según los índices que se muestran en la Tabla 2. Estos futbolistas aceptan positivamente el entrenamiento al que han sido sometidos, manifestando buenas puntuaciones en relación a la diversión, tal y como queda reflejado en el ítem 1 (un 60,8\% afirman haberse divertido mucho con su entrenamiento) y en la pregunta 6 (un 90,2\% apuntan haber disfrutado bastante o mucho). Los datos del ítem 3 refuerzan los resultados anteriores, pues la mayoría de los niños participantes del modelo tradicional niegan haberse aburrido con el tipo de tareas practicadas, $(70,6 \%$, responden nada en el ítem 3).

Según lo que se desprende del ítem 2, estos jugadores vinculados a las tareas de carácter analítico, encuentran interesante el entrenamiento practicado (un 35,3\% lo valora como bastante interesante y un $54,9 \%$ opina que fue muy interesante). Además los sujetos evaluados reconocen haberse involucrado con su programa de intervención, de forma que dentro del ítem 4 "Me meto plenamente en el juego cuando entreno a fútbol", el 84,3\% de los consultados responde haber estado bastante o muy centrado en su tarea. Por último, del análisis de las preguntas 5 y 7 se desprende una buena acogida de este tipo de prácticas: el $82,4 \%$, responden nada en el ítem 5 ("Cuando entreno a fútbol, generalmente deseo que el juego acabe pronto"), mientras que el 72,6\% eligen entre bastante y mucho al preguntarles lo rápido que pasa el tiempo cuando entrenan.

Tabla 2. Resultados del "Cuestionario de diversión con el entrenamiento del fútbol”, en los futbolistas del modelo técnico, expresados en frecuencias y porcentaje.

\begin{tabular}{|c|c|c|c|c|c|}
\hline Items & & Nada & Poco & Bastante & Mucho \\
\hline \multirow{2}{*}{ 1. Me suelo divertir cuando entreno a fútbol } & Frecuencia & 2 & 3 & 15 & 31 \\
\hline & Porcentaje & $3,9 \%$ & $5,9 \%$ & $29,4 \%$ & $60,8 \%$ \\
\hline \multirow{2}{*}{ 2. Suelo encontrar interesante entrenar a fútbol } & Frecuencia & 1 & 4 & 18 & 28 \\
\hline & Porcentaje & $2,0 \%$ & $7,8 \%$ & $35,3 \%$ & $54,9 \%$ \\
\hline \multirow{2}{*}{ 3. Normalmente me aburro cuando entreno a fútbol } & Frecuencia & 36 & 13 & 1 & 1 \\
\hline & Porcentaje & $70,6 \%$ & $25,5 \%$ & $2,0 \%$ & $2,0 \%$ \\
\hline \multirow{2}{*}{ 4. Me meto plenamente en el juego cuando entreno a fútbol } & Frecuencia & 3 & 5 & 21 & 22 \\
\hline & Porcentaje & $5,9 \%$ & $9,8 \%$ & $41,2 \%$ & $43,1 \%$ \\
\hline \multirow{2}{*}{ 5. Cuando entreno a fútbol, generalmente deseo que el juego acabe pronto } & Frecuencia & 42 & 7 & 2 & 0 \\
\hline & Porcentaje & $82,4 \%$ & $13,7 \%$ & $3,9 \%$ &, $0 \%$ \\
\hline \multirow{2}{*}{ 6. Disfruto entrenando a fútbol } & Frecuencia & 5 & 0 & 17 & 29 \\
\hline & Porcentaje & $9,8 \%$ &, $0 \%$ & $33,3 \%$ & $56,9 \%$ \\
\hline \multirow{2}{*}{ 7. Parece que el tiempo pasa volando cuando entreno a fútbol } & Frecuencia & 7 & 7 & 14 & 23 \\
\hline & Porcentaje & $13,7 \%$ & $13,7 \%$ & $27,5 \%$ & $45,1 \%$ \\
\hline
\end{tabular}


Los niños que entrenaron con el programa basado en la táctica, valoran los diferentes del Cuestionario de diversión con el entrenamiento del fútbol, tal y como se observa en la Tabla 3. El entrenamiento construido con juegos tácticos de fútbol ha resultado divertido, puesto que el 68\% de los participantes ha respondido en el ítem 1 ("Me suelo divertir cuando entreno a fútbol") haberse divertido mucho con este tipo de intervención. La buena acogida del programa táctico, queda reflejada también en la pregunta 6, "Disfruto entrenando a fútbol", puesto que un $74 \%$ de los consultados reconoce haber disfrutado mucho con su entrenamiento, mientras que sólo un $4 \%$ reconoce haberse divertido poco o nada con las sesiones construidas con tareas tácticas de fútbol. Estos datos están reforzados con las cifras correspondientes al ítem 3, pues la mayoría de estos sujetos subrayan no haberse aburrido mientras entrenaban a fútbol con actividades que recogían la esencia del deporte (un 2\% afirma haberse aburrido bastante y ningún jugador responde haberse aburrido mucho durante el entrenamiento).

Según las respuestas relativas al ítem 2, "Suelo encontrar interesante entrenar a fútbol", el programa de entrenamiento táctico ha sido calificado como atractivo por el 94\% de los evaluados (el 30\% lo consideran bastante interesante y un $64 \%$ lo valoran como muy interesante). En esta línea, al preguntar en el ítem 4, los jugadores se muestran implicados con la tarea a desarrollar ( $46 \%$ señalan que se han metido mucho en el juego), si bien existe un $10 \%$ de sujetos que también puntúan con el valor mínimo en esta cuestión. Finalmente, el $92 \%$ de los niños responden nada, en la pregunta 5 ("cuando entreno a fútbol, generalmente deseo que el juego acabe pronto") y un alto porcentaje de los consultados creen que el tiempo pasa rápidamente cuando en el ítem 7 se les pregunta por esta cuestión (el 78\% contestan mucho cuando en el ítem 7 , "Parece que el tiempo pasa volando cuando entreno a fútbol").

Tabla 3. Resultados del "Cuestionario de diversión con el entrenamiento del fútbol”, en los futbolistas del modelo táctico, expresados en frecuencias y porcentajes.

\begin{tabular}{|c|c|c|c|c|c|}
\hline Items & & Nada & Poco & Bastante & Mucho \\
\hline \multirow{2}{*}{ 1. Me suelo divertir cuando entreno a fútbol } & Frecuencia & 0 & 2 & 14 & 34 \\
\hline & Porcentaje & $0 \%$ & $4 \%$ & $28 \%$ & $68 \%$ \\
\hline \multirow{2}{*}{ 2. Suelo encontrar interesante entrenar a fútbol } & Frecuencia & 0 & 3 & 15 & 32 \\
\hline & Porcentaje & $0 \%$ & $6 \%$ & $30 \%$ & $64 \%$ \\
\hline \multirow{2}{*}{ 3. Normalmente me aburro cuando entreno a fútbol } & Frecuencia & 39 & 10 & 1 & 0 \\
\hline & Porcentaje & $78 \%$ & $20 \%$ & $2 \%$ & $0 \%$ \\
\hline \multirow{2}{*}{ 4. Me meto plenamente en el juego cuando entreno a fútbol } & Frecuencia & 5 & 1 & 21 & 23 \\
\hline & Porcentaje & $10 \%$ & $2 \%$ & $42 \%$ & $46 \%$ \\
\hline \multirow{2}{*}{ 5. Cuando entreno a fútbol, generalmente deseo que el juego acabe pronto } & Frecuencia & 46 & 2 & 1 & 1 \\
\hline & Porcentaje & $92 \%$ & $4 \%$ & $2 \%$ & $2 \%$ \\
\hline \multirow{2}{*}{ 6. Disfruto entrenando a fútbol } & Frecuencia & 2 & 0 & 13 & 35 \\
\hline & Porcentaje & $4 \%$ & $0 \%$ & $26 \%$ & $74 \%$ \\
\hline \multirow{2}{*}{ 7. Parece que el tiempo pasa volando cuando entreno a fútbol } & Frecuencia & 1 & 1 & 9 & 39 \\
\hline & Porcentaje & $2 \%$ & $2 \%$ & $18 \%$ & $78 \%$ \\
\hline
\end{tabular}

Los datos representados en la Figura 1, detallan que el grupo de futbolistas que participó en las sesiones del programa táctico, otorga puntuaciones más elevadas en todas las preguntas del Cuestionario de diversión con el entrenamiento del fútbol. Esto puede comprobarse a partir de las cifras vinculadas al indicador de frecuencia de los diferentes ítems para cada uno de los programas de entrenamiento. Una comparación de los resultados correspondientes al ítem 1 refleja que los jugadores que entrenan mediante juegos de aplicación táctica, se divierten más que los que completaron las sesiones basadas en ejercicios de tipo analítico. En cuanto al grado de disfrute asociado a cada programa, los datos de la pregunta 6 conservan la ventaja del programa centrado en tareas de aplicación táctica. Por otra parte, según lo indicado en el ítem 3, los niños del grupo técnico parece que se aburren más que sus compañeros participantes del modelo cognitivo. Observando los datos correspondientes a la pregunta 2, advertimos que los dos programas han despertado el interés en los participantes, pero son los futbolistas del programa táctico los que encuentran más interesante sus sesiones de trabajo. Todos los grupos manifiestan su compromiso con el entrenamiento en términos de concentración, sin que podamos reflejar una diferencia importante en las respuestas a la pregunta 4 ("Me meto plenamente en el juego cuando entreno a fútbol"). La similitud entre resultados se repite en el ítem 5, de forma que los sujetos, con independencia del programa desarrollado no 
tienen especial preocupación por terminar las sesiones de entrenamiento. Finalmente, al analizar la cuestión 7 "El tiempo pasa volando cuando entreno a fútbol", se observa de nuevo un diferencia favorable al modelo táctico, que remarca como en estos jugadores el tiempo invertido trascurre más rápido que en los futbolistas del modelo tradicional.

Figura 1. Representación del valor "indicador sintético de frecuencia”, para los ítems del "Cuestionario de diversión con el entrenamiento del fútbol”, según modelo de entrenamiento.

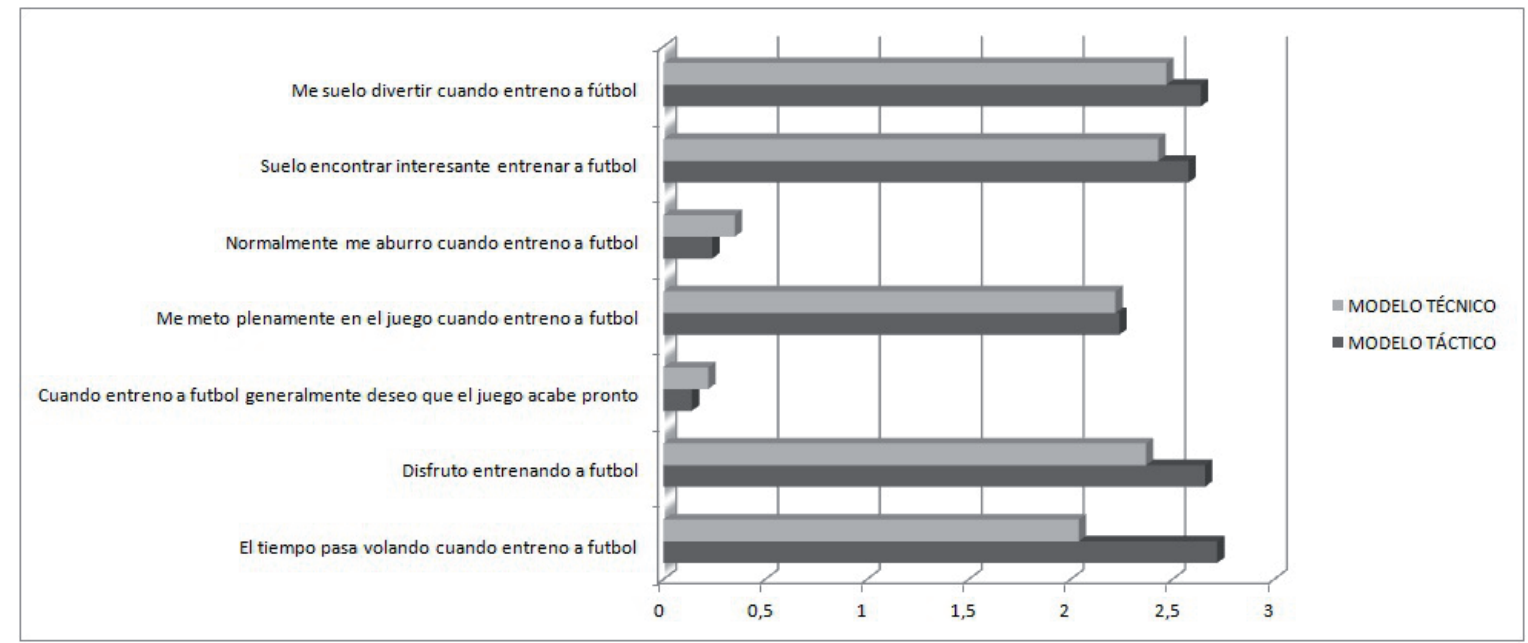

Aplicando la prueba $t$ para muestras independientes tal y como se muestra en la Tabla 4, comprobamos que no existe una ventaja estadísticamente significativa de un tipo de entrenamiento en relación a la diversión en los sujetos del estudio. Esta ausencia de contrastes importantes se manifiesta en cada uno de los ítems empleados, aunque con dos excepciones reveladoras. En el ítem 6 se aprecia una tendencia favorable al entrenamiento táctico, que sin llegar a ser significativa $(\mathrm{p}=$ $0,060)$, indica una predisposición a disfrutar más con el entrenamiento basado en los juegos de aplicación táctica. La comparación de los resultados obtenidos en la pregunta 7 (" $E l$ tiempo pasa volando cuando entreno a fútbol") manifiesta una diferencia altamente significativa a favor del grupo entrenado con tareas de fútbol reducido.

Tabla 4. Comparación del valor "Indicador Sintético de Frecuencia”(Media (m) y Desviación Estándar (Sd)), referido a las diferentes categorías de respuesta del "Cuestionario de diversión con el entrenamiento del fútbol”, a través del prueba t para muestras independientes. ${ }^{*} \mathrm{p} \leq 0,05 ;{ }^{*} \mathrm{p} \leq 0,01$.

Items

1. Me suelo divertir cuando entreno a fútbol 2. Suelo encontrar interesante entrenar a futbol 3. Normalmente me aburro cuando entreno a futbol 4. Me meto plenamente en el juego (o en la acción) cuando entreno a futbol

5. Cuando entreno a futbol generalmente deseo que el juego acabe pronto

6. Disfruto entrenando a futbol

7. El tiempo pasa volando cuando entreno a futbol
Indicador Sintético de Frecuencia

2,47

2,43

0,35

2,22

0,22

2,37

2,04

\begin{tabular}{lll} 
NICO & \multicolumn{2}{c}{ M. TÁCTCIO } \\
Sd & $m$ & Sd \\
0,784 & 2,64 & 0,563 \\
0,728 & 2,58 & 0,609 \\
0,627 & 0,24 & 0,476 \\
0,856 & 2,24 & 0,916 \\
& & \\
0,503 & 0,14 & 0,535 \\
0,916 & 2,66 & 0,557 \\
1,076 & 2,72 & 0,607
\end{tabular}

Valor $p$ de significación estadística a partir de la Prueba $t$ para muestras independientes

0,215

0,269

0,311

0,891

0,465

$0,557 \quad 0,060$

$0,6070,000^{* *}$

\section{Discusión}

Los resultados obtenidos en la presente investigación, indican que la metodología que tiene en consideración la lógica interna del fútbol, es más atrayente que aquellas otras que se estructuran a partir de situaciones parciales y descontextualizadas de la realidad del juego. Parece que los sujetos que entrenaron con los juegos de aplicación táctica, tienden 
a disfrutar más con su entrenamiento. Entendiendo que los jóvenes utilizan indistintamente diversión y disfrute (Garn y Cothran, 2006; Scanlan y Simons, 1992), podemos afirmar que el programa táctico será más divertido.

Los estudios de Allison y Thorpe (1997), Duran y Lasierra (1987), Griffin, Oslin y Mitchell (1995), Méndez (1999) y Turner (1996), ya indicaron que un enfoque comprensivo del deporte, a través de una orientación basada en la táctica, donde el juego es el recurso estrella del aprendizaje, genera más diversión que otro tratamiento incrustado en la técnica, basado en ejercicios de tipo analítico para la mejora exclusiva del mecanismo de ejecución.

Aunque parece asumido que para deleitarse con el entrenamiento de un deporte, es recomendable utilizar el juego como recurso protagonista dentro de las sesiones (Arias, 2008; Duran y Lasierra, 1987; García, 2001; Ponce, 2006; Torres, 2005; Turner y Martinek, 1992; Valero, 2005), las puntuaciones registradas en cada grupo, no determinan un efecto excluyente entre metodologías, puesto que con ambos programas los jóvenes futbolistas han experimentado un elevado índice de diversión. Esto contrasta con lo señalado por Devis y Peiró (1995), quienes afirman que en el ámbito del aprendizaje deportivo, para lograr una práctica divertida es obligado prescindir de las tradicionales intervenciones basadas en la técnica. Según lo que se desprende de los resultados obtenidos en nuestro estudio, no es posible descartar ninguna de las estrategias metodológicas empleadas, si bien tal y como sucede en el trabajo de Ponce (2006), no podemos olvidar, que dentro de la semejanza entre las opiniones derivadas de la aplicación de dos estilos de enseńanza-entrenamiento opuestos, disfrutan más aquellos que desarrollan el modelo táctico.

Con respecto al interés provocado por los programas, la muestra del estudio manifiesta haber encontrado interesante los tipos de entrenamiento desarrollados. Si bien las puntuaciones vuelven a resultar levemente favorables a los sujetos que componen el grupo preparado a través del juego; el entrenamiento analítico, también parece despertar el interés de los jugadores que lo desarrollan. Por esta razón, y dada la vinculación que puede existir entre lo interesante y lo divertido, se reafirma el disfrute generado en los nińos por la intervención táctica y técnica.

En el cuestionario, también se intenta diagnosticar el nivel de diversión asociado al entrenamiento, consultando aspectos relacionados con el deseo de finalizar el entrenamiento y lo rápido que pasa el tiempo de sesión. Únicamente se ha encontrado una distancia considerable entre las opiniones en este último tema de consulta. La diferencia favorable al grupo participante en el programa táctico, reflejaría una mayor diversión asociada a este tipo de entrenamiento y subrayaría una mayor motivación en estos jugadores. Según Méndez (1999), todas aquellas estrategias que convierten al sujeto en un agente activo de su práctica, son las más adecuadas desde el punto de vista de la motivación. Por esta razón, nuestro programa de entrenamiento táctico, que es el que concede más libertad al jugador para resolver los problemas derivados de su intervención sería el que más aumenta la motivación. Este factor es elemental, ya que una mayor amotivación provocaría niveles de diversión más bajos en los sujetos (Guay et al., 2000; Carratalá et al., 2006).

Por último, debido a la consideración semejante de los dos planteamientos metodológicos, los niños valoran positivamente la práctica del fútbol en su conjunto, sin analizar el tipo de intervención al que han podido ser sometidos. La mayoría entienden que van a practicar el deporte que les gusta y que han elegido voluntariamente, razón suficiente para que les divierta. En el futuro, para reafirmar los datos, se podría intentar conocer la opinión de un mismo grupo, sometido a los dos tipos de intervención.

Podemos concluir que tanto el programa de entrenamiento técnico, como el modelo construido con juegos de aplicación táctica, han resultado suficientemente divertidos para los niños participantes en el estudio. No obstante, existen leves diferencias a favor de la intervención táctica, que confirman la conveniencia del juego como herramienta especialmente propicia para generar contexto de aprendizaje positivos, que aumenten en lo posible la adherencia del niño en el deporte. Las tareas de tipo mecánico, muy implicadas en el desarrollo del aprendizaje por imitación, que se dirigen a potenciar el contenido técnico, aún siendo divertidas, se hacen menos llevaderas para los participantes, puesto que tal y como reconocen los sujetos implicados en esta intervención, el tiempo de entrenamiento transcurre con mayor lentitud, en comparación con lo rápido que pasa cuando se practica bajo el modelo táctico. Por esta razón, los ejercicios técnicos propios del modelo de enseńanza-entrenamiento tradicional, hacen que el niño desee con más ganas que el entrenamiento finalice. Esta sensación no sucede en los niños entrenados por medio de la estrategia global, quedando confirmado que son estos jugadores los que más han disfrutado con el entrenamiento.

\section{Referencias}

1. Allison, S. y Thorpe, R. (1997). A comparison of the effectiveness of two approaches to teaching games within physical education. A skills approach versus a games for understanding approach. The British Journal of Physical Education, 28 (3), 9-13.

2. Alonso, M. y Lago, C. (2009). Planificación de los contenidos técnico- tácticos en categorías de formación. En L. Casáis, E. Domínguez y C. Lago, (eds.) Futbol base: el entrenamiento en categorias de formación (pp. 37-95). MC Sports.

3. Arias, J. L. (2008). El proceso de formación deportiva en la iniciación a los deportes colectivos fundamentado en las características del depor- 
tista experto. Retos. Nuevas tendencias en Educación Física, Deporte y Recreación, 13, 28-32.

4. Blázquez, D. y Batalla, A. (1995). La edad de iniciación: vísteme despacio que tengo prisa. En D. Blázquez, (Dir.) Iniciación deportiva y deporte escolar (pp. 115-130). Barcelona. Inde.

5. Cárdenas, D. (2006). El proceso de formación táctica colectiva en el baloncesto desde la perspectiva constructivista. Lecturas: educación física y deportes, Revista Digital, 94. Extraído el 15 septiembre de 2011 desde http://www.efdeportes.com/efd94/balonces.htm.

6. Carratalá, E., Guzmán, J.F., Carratalá, V. y García, A. (2006). La diversión en la práctica deportiva en función del modelo jerárquico de la motivación: un estudio con deportistas de especialización deportiva. Revista Motricidad, 15, 148-155.

7. Cervelló, E. (1996). La motivación y el abandono deportivo desde la perspectiva de las metas de logro. Tesis Doctoral. Universidad de Valencia.

8. Cervelló, E., Escartí, A. y Balagué, G. (1999). Relaciones entre la orientación de metas disposicional y la satisfacción con los resultados deportivos, creencias sobre las causas de éxito en deporte y la diversión con la práctica deportiva. Revista de Psicología del Deporte, 11, 41-57.

9. Contreras, O.R., De la Torre, E. y Velázquez, R. (2001). Iniciación deportiva. Madrid. Síntesis.

10. Devis, J. y Peiró, C. (1995). Enseńanza de los deportes de equipo: la comprensión en la iniciación de los juegos deportivos. En D. Blázquez, (Dir.) La iniciación deportiva y el deporte escolar (pp. 333-350). Barcelona. Inde.

11. Duda J.L. y Nicholls, J.G. (1992). Dimensions of achievement motivation in schoolwork and sport. Journal of Educational Psychology, 84 (3), 290-299.

12. Duran, C. y Lasierra, G. (1987). Estudio experimental sobre didáctica aplicada a la iniciación de los deportes colectivos. Revista metodológica de investigación y documentación sobre las ciencias de la educación física y el deporte, 7, 92-128.

13. García, J. A. (2001). Adquisición de la competencia para el deporte en la infancia: el papel del conocimiento y la comprensión en la toma de decisiones en balonmano. Tesis Doctoral. Universidad de Extremadura.

14. Garn, A.C. y Cothran, D.J. (2006). The fun factor in physical education. Journal of teaching in physical education, 25, 281-297.

15. Griffin, L.L., Oslin, J.L. y Mitchell, S.A. (1995). An analysis of two instructional approaches to teaching net games. Research Quarterly for Exercise and Sport, 66 (Suppl.), A-64.

16. Guay, F., Vallerand, R.J., y Blanchard, C.M. (2000). On the assesment of state intrinsic and extrinsic motivation: The Situational Motivation Scale (SMS). Motivation and Emotion, 24, 175-213.

17. Lasierra, G. (1990). Aproximación a una propuesta de aprendizaje de los elementos tácticos individuales en los deportes de equipo. Apunts. Educación física y deportes, 24, 59-68.

18. Martínez Chávez, H. F. (2001). Fútbol: caracterización de los modelos de enseñanza. Una oportunidad para el aprendizaje significativo. Lecturas: educación física y deportes, Revista Digital, 36. Extraído el 30 de junio de 2011 desde http://www.efdeportes.com/efd36/futbols.htm.
19. Méndez, A. (1999). Análisis comparativo de las técnicas de enseñanza en la iniciación a dos deportes de invasión. Tesis Doctoral. Universidad de Granada.

20. Molinero, O., Salguero, A., Tuero, C., Álvarez, E. y Márquez, S. (2006). Dropout from youth sports: relationships to gender, type of sport and level of competition. Journal of sport behavior, 29 (3), 255-269.

21. Ponce, F. (2006). La eficacia de la utilización de una técnica de enseñanza mediante indagación o búsqueda en la mejora de diferentes aspectos funcionales, técnico-tácticos, decisionales y motivacionales, en futbolistas de categoría alevín, frente a una intervención tradicional y su transferencia a la competición. Tesis Doctoral. Universidad de Granada.

22. Sánchez, F. (1995). El deporte como medio formativo en el ámbito escolar. En D. Blázquez. (Dir.) Iniciación deportiva y deporte escolar (pp. 77-93). Barcelona. Inde.

23. Scanlan, T.K., y Simons, J.P. (1992). The construct of sport enjoyment. En G.C. Roberts (Ed.) Motivation in sport and exercise. Champaign, Il. Human Kinetics.

24. Soares, F. y Santana, W. C. (2005). Iniciaçao ao futsal: crianzas jogam para aprender ou aprendem para jogar? Lecturas: educación fisica y deportes, Revista Digital, 85. Extraído el 30 de junio de 2011 desde http:// efdeportes.com/efd85/futsal.htm.

25. Thorpe, R. (1992). La comprensión en el juego de los niños: una alternativa a la enseńanza de los juegos deportivos. En. J. Devís y C. Peiró (Eds.), Nuevas perspectivas curriculares en educación física: la salud y los juegos modificados. (pp. 185-208). Barcelona. Inde.

26. Torres, J. (2004). Nuevas perspectivas en el proceso de enseñanza-aprendizaje en las escuelas multideportivas. IV Jornadas sobre el Deporte Base. Generalitat Valenciana, Secretaria Autonómica del deporte.

27. Torres, J. (2005). Un nuevo marco didáctico para el deporte educativo en la sociedad postmoderna. Del romanticismo deportivo del XIX a regreso al futuro del XXI. V Jornadas sobre el Deporte Base. Generalitat Valenciana, Secretaria Autonómica del deporte.

28. Turner, A. y Martinek, T. J. (1992). A comparative analysis of two models for teaching games. International Journal of Physical Education, 29 (4), 15-31.

29. Turner, A. (1996). Teaching for Understanding. Myth or reality? Journal of Physical Education, Recreation and Dance, 67 (4), 46-48/55.

30. Valero, A. (2003). Comparación de los efectos de dos modelos de iniciación para la enseñanza aprendizaje de tres disciplinas de atletismo. Tesis Doctoral. Universidad de Jaen.

31. Valero, A. (2005). Análisis de los cambios producidos en la metodología de la iniciación deportiva. Apunts. Educación Física y Deportes. 79, 59-67.

32. Valero, A., Conde, A., Delgado, M. y Conde, J.L. (2004). Construcción y validación de un cuestionario de diversión y adherencia hacia la práctica del atletismo en la ecuación primaria. Revista Española de Educación Física y Deportes 1, 119-130.

33. Valero, A. y Latorre, P. A. (1998). La motivación en la iniciación deportiva. En A. García, J. F. Ruiz y A. J. Casimiro (Eds.). La enseñanza de la Educación Física y el deporte escolar (153-160). Málaga. Junta de Andalucía.

34. Wein, H. (1995). Fútbol a la medida del niño. Madrid. CEDIF. 